\title{
Haptic aftereffect of curved surfaces
}

Ingrid M L C Vogels, Astrid M L Kappers, Jan J Koenderink

Helmholtz Instituut, Princetonplein 5, 3584 CC Utrecht, The Netherlands

Received 15 May 1995, in revised form 14 November 1995

\begin{abstract}
A haptic aftereffect of curved surfaces is demonstrated. Two spherical surfaces were presented sequentially to human subjects. They rested one hand on the first (conditioning) surface. After a fixed conditioning period they transferred their hand to the second (test) surface and judged whether the test surface was convex or concave. In experiment 1 the curvature of the conditioning surface was varied; the subject's judgment of convexity or concavity of the test surface was strongly shifted in the direction opposite to the curvature of the conditioning surface (negative aftereffect). Therefore, subjects judged a flat surface to be concave after being exposed to a convex surface. After a conditioning period of $5 \mathrm{~s}$ the shift was about $20 \%$ of the curvature of the conditioning surface. In experiment 2 the duration of the conditioning period was varied; the magnitude of the aftereffect could be described by a first-order integrator with a time constant of $2 \mathrm{~s}$. In experiment 3 the time interval between the conditioning period and the touching of the second surface was varied; the magnitude of the aftereffect could be described by an exponential decay with a time constant of $40 \mathrm{~s}$. It is concluded that the haptic aftereffect of curved surfaces is an important effect that occurs almost instantaneously and lasts for an appreciable period.
\end{abstract}

\section{Introduction}

Probably all modalities give rise to aftereffects. Gibson (1937) was one of the first to emphasise this similarity between the senses. In his paper he described several examples of visual, tactual, and gustatory stimuli which lead to adaptation with negative aftereffects. Adaptation and aftereffect, both of which are caused by prolonged exposure to a constant stimulus, are in fact two distinct phenomena. Adaptation affects the perception of the stimulus itself. Aftereffects are defined as a change of the 'physicalphenomenal correspondence' of the stimulus dimension occurring after prolonged stimulation. The term adaptation is often used as a synonym for various ill-defined terms such as normalisation, satiation, habituation, or fatigue. Such careless usage often leads to unfortunate misunderstandings. For instance, Gibson (1937) assumed that adaptation and aftereffect are two facets of a single process, whereas Colheart (1971) argued that they may be quite separate effects. In this paper we use the mentioned conventions.

In modalities such as vision or hearing, adaptation and aftereffects have been investigated intensively. In haptic perception, which relies on both the cutaneous sense and kinesthesis, these phenomena have received considerably less attention, although they have already been known phenomenologically for a long time. Here a number of examples of studied haptic aftereffects is presented. We certainly do not aim to be complete.

It is well known that tepid water feels cold to a hand previously exposed to hot water. Abbott (1914) mentioned that the phenomenal 'neutral' temperature appears to be so adaptable that it can be raised as high as $39{ }^{\circ} \mathrm{C}$, whereupon $37^{\circ} \mathrm{C}$ feels cold and it can fall as low as $11^{\circ} \mathrm{C}$, whereupon $12^{\circ} \mathrm{C}$ feels warm. Thalman (1922) applied a cord of rough material to the underside of the bare forearm of human subjects. The cord moved in a constant direction and with a constant velocity. As soon as the movement stopped, subjects perceived a movement in the opposite direction both when the cord was removed from the arm and when the cord remained in contact with 
the arm. Wohlgemuth (1911) and Hazlewood (1971) failed to find evidence in support of a tactile movement aftereffect. Recently, Hollins and Favorov (1994) demonstrated that the texture of the surface applied to the skin might determine the occurrence or nonoccurrence of the aftereffect. When subjects cupped their hand around a moving drum which was covered with a smooth microtexture combined with a square wave of low spatial frequency, the aftereffect was especially effective. The vividness and duration of the aftereffect increased over the range of adaptation durations explored $(30-180 \mathrm{~s})$. Gibson (1933) found that after $3 \mathrm{~min}$ of moving the fingers over a curved cardboard edge a straight edge felt curved in the opposite direction. Nafe and Wagoner (1941) investigated pressure adaptation. They showed that tactile sensation produced by a weight resting on the skin disappeared when the rate at which the weight sank into the skin had decreased below a minimum rate required to stimulate. The removal of the weight produced a clear tactile sensation. Koehler and Dinnerstein (1947) showed that when the width of a test bar felt with one hand had to be matched with the other hand, the matching depended on a satiation bar previously felt with the tested hand. A satiation object smaller than the test object enlarged the matched width of the test bar; a wider satiation object had the opposite effect. Gibson (1963) investigated the aftereffect of the perception of vergence. He found that two parallel palm boards felt divergent after they had been held convergent for some time, and felt convergent after being held divergent. In order to make the surfaces feel parallel they had to be rotated over about $5^{\circ}$ to $10^{\circ}$, according to the amount of convergence or divergence of the hand during adaptation. After an adaptation period of $12 \mathrm{~s}$ the aftereffect did not show any increase with time and after a so-called recovery period of $56 \mathrm{~s}$ the aftereffect still had no tendency to decrease. Hahn (1966) investigated vibrotactile adaptation by two different methods. In the first method he measured the absolute threshold, which is the just-noticeable amplitude of the sinusoidal vibration. In the second method he measured the subjective magnitude, by matching the sensation in the adapted finger to that in the contralateral finger. Increasing the time of vibrotactile stimulation resulted in an increase of the threshold and a decrease of the subjective magnitude. Both methods showed the same temporal course of adaptation. Adaptation continued to increase after a period of $20 \mathrm{~min}$.

Not only simple aftereffects, like previous examples, but also contingent aftereffects have been demonstrated in the haptic modality. In contingent aftereffects, a correlation between two stimulus dimensions is established during an inspection period, and during a subsequent test period the appearance of an object on one dimension is dependent on the location on the other dimension. If a narrow inspection block, located on the subject's left, and a wide block, located on the right, are alternately grasped by a subject between the fingers of a single hand then an intermediate test block presented on the left is perceived wider than an equal block on the right (Walker and Shea 1974). The perceived width of the test block is contingent on the location of the block. If a rectangular inspection block is oriented with its long side horizontally and with its short side vertically, and if a subject alternately grasps this block horizontally and vertically, then the horizontal side of a subsequently presented square test block is perceived shorter than the vertical side (Walker 1977). Here the perceived size of a test block is contingent on the orientation of the block.

Thus, many haptic stimuli give rise to an aftereffect. Because the haptic sense is particularly important for the recognition and manipulation of objects, it would be useful if the haptic sense provided us with veridical information about the shape and curvature of environmental objects. Because aftereffects could affect veridicality, it would be of interest to know whether aftereffects occur when subjects touch curved three-dimensional objects. Such an aftereffect of curved surfaces has not been reported in the literature. 
It is known that in general the categorisation of curvature of one-dimensional strips is not veridical. Hunter (1954) and Davidson (1972) let subjects judge whether strips were convex, concave, or straight. All subjects judged the straight strips to be curved and the strips which were judged to be straight typically corresponded to a curved strip. So, straight was not judged to be straight. It is conceivable that in the case of the judgment of curved surfaces, the surface which is judged to be flat (phenomenal flatness) also corresponds to a curved surface. The interesting question is, however, whether the phenomenal flatness, veridical or not, is constant over time. The experiments reported here are designed to investigate this. In the first experiment we investigated whether the categorisation of curvature is influenced by a previously touched curved surface. Two spherical surfaces (a conditioning surface and a test surface) were presented sequentially. We measured which curvature of the test surface was judged to be flat for several differently curved conditioning surfaces. We found an aftereffect; the phenomenal flatness depended on the curvature of the conditioning surface. In experiment 2 we investigated how much time was needed to build up the aftereffect. The magnitude of the aftereffect was measured for several different periods of time during which the conditioning surface was touched (conditioning period). In experiment 3 we investigated whether the aftereffect was maintained or whether it disappeared after the touching had ceased. The magnitude of the aftereffect was measured for several time intervals between the conditioning period and the touching of the test surface.

\section{Experiment 1}

In the first experiment we tested whether judgments of convexity or concavity of a spherical surface are influenced by the curvature of a previously touched surface. The results of this experiment can give us an idea of how haptic curvature information is processed. Does the haptic system possess an absolute internal reference for curvature or does recalibration occur? The results are also important for other haptic experiments: does it make sense to let subjects judge the absolute curvature when aspects of haptic curvature perception are being investigated?

\subsection{Method}

2.1.1 Experimental setup. The stimuli had a spherical upper surface, either convex or concave, and a flat bottom which rested on a table (see figure 1). The diameter of the stimuli was $20 \mathrm{~cm}$ and the total height ranged between 2.5 and $6.5 \mathrm{~cm}$. This height was not directly related to the curvature, because we randomised the base height.

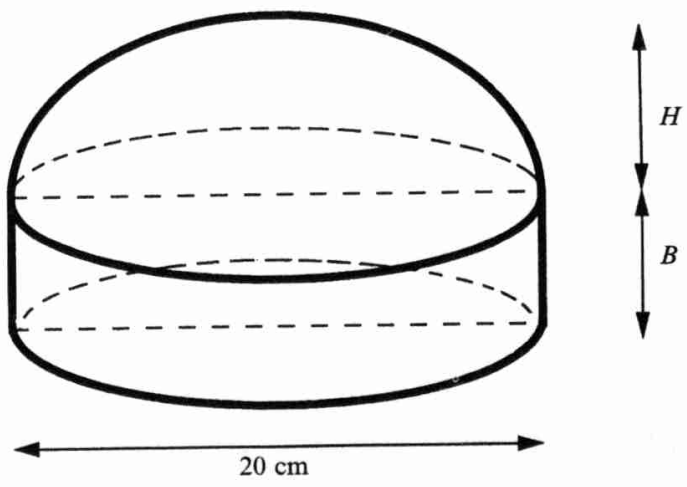

Figure 1. Example of a convex spherical stimulus. All stimuli had a flat bottom and a diameter of $20 \mathrm{~cm}$. The height difference $H$ between the total height of the stimulus and the base height $B$ was $2 \mathrm{~cm}$ for the largest curvature $\left(4 \mathrm{~m}^{-1}\right)$ and $0.125 \mathrm{~cm}$ for the smallest curvature $\left(0.25 \mathrm{~m}^{-1}\right)$. The total height ranged between 2.5 and $6.5 \mathrm{~cm}$. 
The curvature ranged from $-4 \mathrm{~m}^{-1}$ to $4 \mathrm{~m}^{-1}$ and two successive curvatures differed by a factor $\sqrt{2}$ (the whole set of curvatures being $-4,-2.8,-2,-1.4,-1,-0.7,-0.5$, $-0.35,-0.25,0,0.25,0.35,0.5,0.7,1,1.4,2,2.8$, and $\left.4 \mathrm{~m}^{-1}\right)$. Curvature is conventionally expressed as reciprocal radius, so a sphere with radius $2 \mathrm{~m}$ has a curvature of $0.5 \mathrm{~m}^{-1}$. A plane has zero curvature and the curvature of the tip of a needle would tend to infinity. A positively curved surface is called convex, a negatively curved surface is called concave.

2.1.2 Subjects. Three naïve paid subjects, about 20 years old, participated in this experiment; one was a strongly left-handed female (GD) and two were strongly righthanded males (MH and EK). The degree of left/right-handedness is defined by Coren (1993). None of the subjects reported any haptic deficiencies.

2.1.3 Procedure. Subjects were seated behind a curtain in front of a table. They put their right hand under the curtain so that they could touch the stimuli without seeing them. In each trial subjects put their hand on a surface (conditioning surface) for a fixed conditioning period of $5 \mathrm{~s}$. After that period they transferred their hand to a second surface (test surface). Subjects had to decide whether this second surface was convex or concave. They were not allowed to move their hand over the surfaces. All stimuli were in the same position with respect to the thorax when they were touched. Seven conditioning surfaces were used, with curvatures of $-4,-2,-1,0,1,2$, and $4 \mathrm{~m}^{-1}$. In order to measure the phenomenal flatness we presented each conditioning surface together with nine differently curved test surfaces. Each combination of conditioning surface and test surface was presented 15 times. The total number of presentations was thus 945 ( 7 conditioning surfaces $\times 9$ test surfaces $\times 15$ presentations). All presentations were randomly distributed over seven sessions. Each session took about $1 \mathrm{~h}$, so for each subject the experiment involved $7 \mathrm{~h}$ in total.

We adapted the range of test surfaces to the curvature of the surface judged to be flat. This was done by means of a pilot experiment in which we estimated which surface was judged to be flat for each conditioning surface and each subject. The nine test surfaces were chosen symmetrically around this phenomenal flat surface. In this way the range of test surfaces was optimised for each conditioning surface.

\subsection{Results}

In figure 2 we present the results in the case of a flat conditioning surface $\left(0 \mathrm{~m}^{-1}\right)$ for subject GD. The percentage of convex judgments is plotted against the curvature of the test surface. The psychometric function is fitted with an error function, according to the Levenberg-Marquardt method (Press et al 1988). This method minimises $\chi^{2}$. Figure 2 is a typical example of how well the function fits the data $\left(\chi^{2}=3642\right)$. The fit can be characterised by two parameters: the shift and the threshold. The shift corresponds to the value of the $50 \%$ point. In our analysis the shift of the psychometric curve is called 'phenomenal flatness' because the curved surface for which convex and concave judgments are equally frequent is assumed to be perceived as flat. The threshold, in this case the threshold of curvature detection, is inversely proportional to the steepness of the curve and corresponds to the difference between the values of the $50 \%$ and $85 \%$ points. Because the psychometric curve is symmetrical, the threshold also corresponds to the difference between the values of the $50 \%$ and $15 \%$ points.

It can be seen that in figure 2 the value of the $50 \%$ point is not zero. In this example, the surface which is perceived as flat corresponds to a geometrically concave surface. For all three subjects phenomenal flatness corresponded to a surface with negative curvature. 


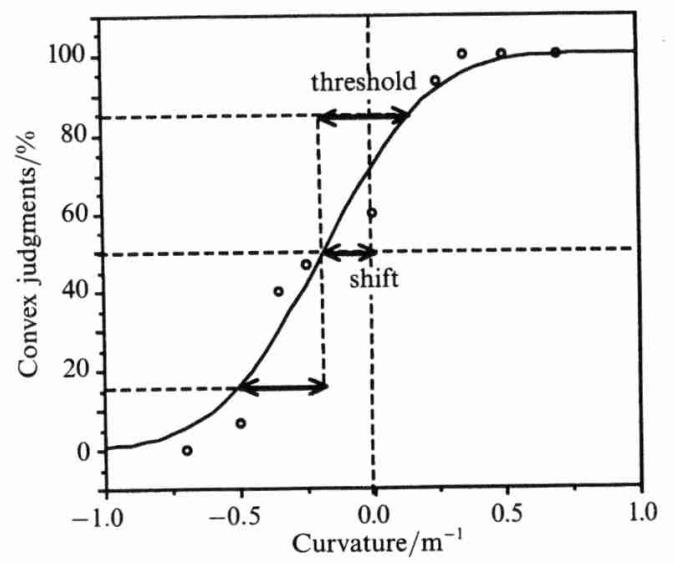

Figure 2. The percentage of convex judgments for a flat conditioning surface as a function of the curvature of the test surface, for subject GD. A psychometric function is fitted to the data points $\left(\chi^{2}=3642\right)$. The shift, which corresponds to the value of the $50 \%$ point, is $-0.18 \mathrm{~m}^{-1}$. This indicates that a geometrically flat surface is not judged to be flat. The threshold for curvature detection, which corresponds to the difference between the values of the $50 \%$ and $85 \%$ (or $15 \%$ ) points, is $0.32 \mathrm{~m}^{-1}$.

Figure 3 shows the results in the case of all conditioning surfaces for subject GD. For clarity the fitted curves are plotted without the data points. Most values of $\chi^{2}$ are somewhat smaller than the value of figure 2; $\chi^{2}$ varies between 105 and 16323 for subject GD, 260 and 7650 for subject $\mathrm{MH}$, and 740 and 4480 for subject EK. The shapes of the psychometric curves look rather similar. Although it seems as if the steepness of the curves decreases with increasing curvature, this is not significant. For the other two subjects the steepness did not systematically increase or decrease. The threshold is thus independent of the curvature of the conditioning surface. The average thresholds are $0.25 \pm 0.04 \mathrm{~m}^{-1}, 0.24 \pm 0.03 \mathrm{~m}^{-1}$, and $0.21 \pm 0.04 \mathrm{~m}^{-1}$ for subjects GD, MH, and EK respectively.

The position of the curves, ie the value of the phenomenal flatness, is not constant. The curves are horizontally shifted with respect to each other, the order being the same

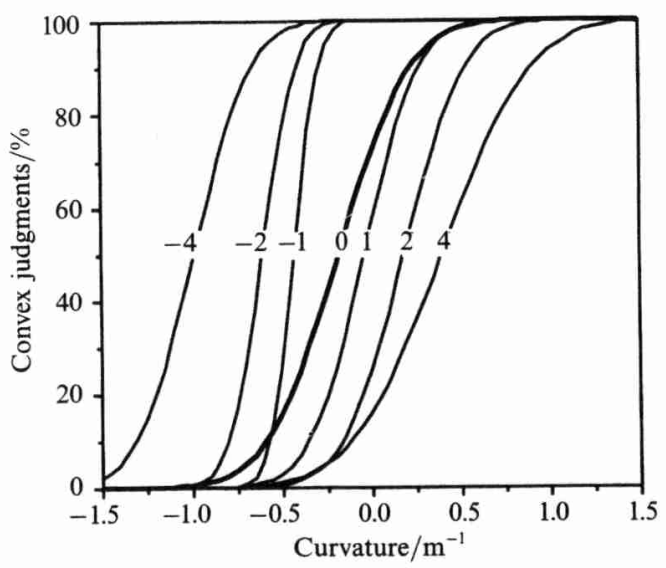

Figure 3. Psychometric curves for seven differently curved conditioning surfaces $(-4,-2,-1,0$, 1,2 , and $4 \mathrm{~m}^{-1}$ ) for subject GD. The values of $\chi^{2}$ are, from left to right, 105, 593, 322, 3642, 1469,3567 , and 16323. The thresholds of the seven curves are almost similar; the average is $0.25 \pm 0.04 \mathrm{~m}^{-1}$. The values of the phenomenal flatness are clearly different; the stronger the conditioning curvature the larger the shift of the phenomenal flatness. This indicates that phenomenal flatness depends on previous exposure to curved surfaces. 
as the order of the curvatures of the conditioning surfaces. The stronger the curvature of the conditioning surface the larger the shift of the phenomenal flatness. When the conditioning surface is convex, phenomenal flatness is shifted towards convex. In other words, a geometrically convex surface is judged to be flat and a geometrically flat surface is judged to be concave. When the conditioning surface is concave, phenomenal flatness is shifted towards concave, so a geometrically flat surface is judged to be convex. Phenomenal flatness depends thus rather critically and systematically on previous exposure to curved surfaces.

In figure 4 the values for the phenomenal flatness of the seven psychometric curves are plotted against the curvature of the conditioning surface (also called conditioning curvature $C$ ), for all three subjects. Apparently, the phenomenal flatness and the conditioning curvature are linearly related in the range of -4 to $4 \mathrm{~m}^{-1}$. A linear relation $P(C)=P_{0}+\alpha C$ describes the data rather well. The offset $P_{0}$ indicates that in the case of a flat conditioning surface the phenomenal flatness is $P_{0} \mathrm{~m}^{-1}$. For curved conditioning surfaces the phenomenal flatness shifts over a distance of $\alpha$ times the conditioning curvature. The fitted relations are $-0.23+0.18 C,-0.41+0.18 C$, and $-0.15+0.15 C$ for subjects $\mathrm{GD}, \mathrm{MH}$, and $\mathrm{EK}$ respectively.
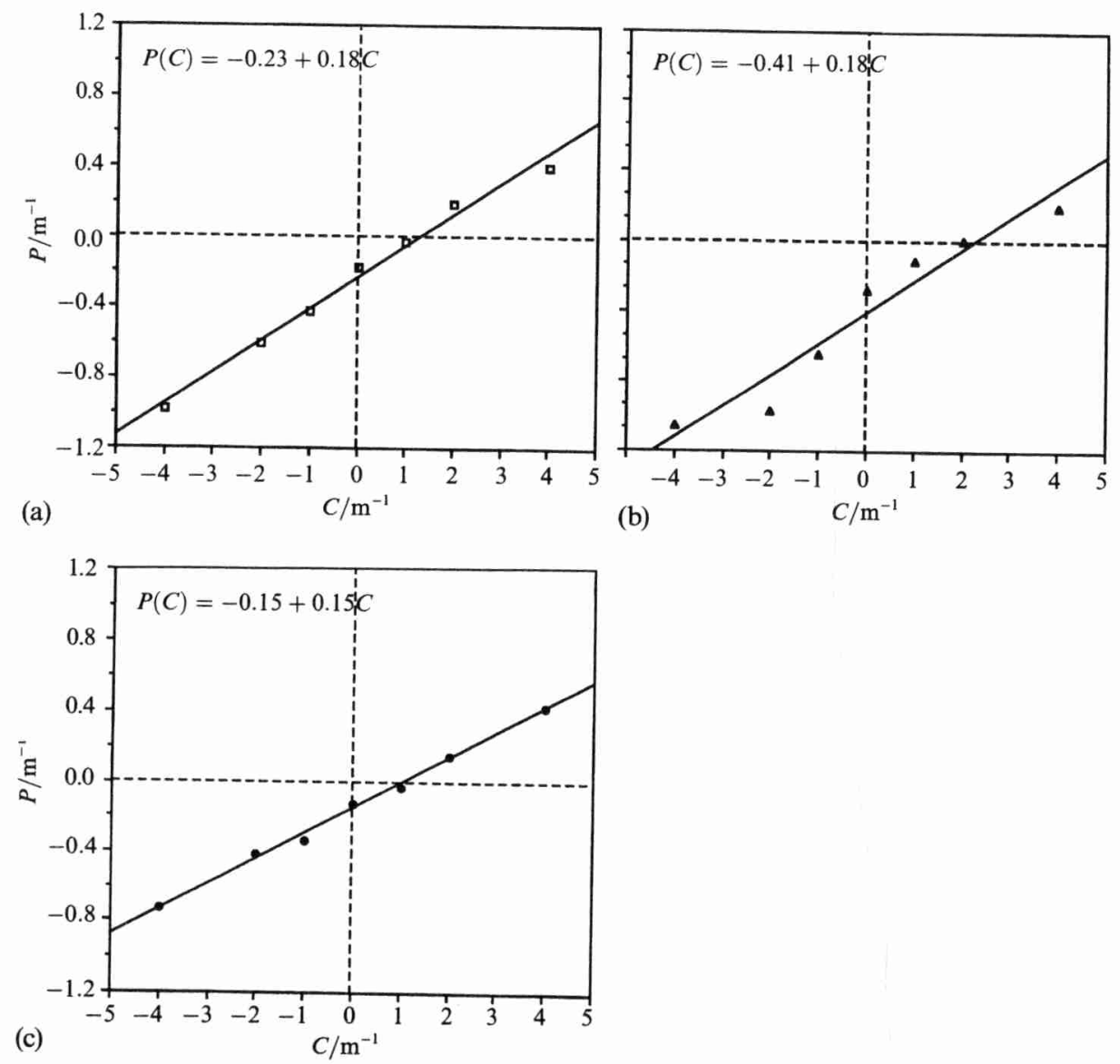

Figure 4. The relation between conditioning curvature $(C)$ and phenomenal fiatness $(P)$, for all three subjects. A linear function is fitted to the data points: $P(C)=P_{0}+\alpha C$. The offset $P_{0}$ corresponds to the phenomenal flatness in the case of a flat conditioning surface. The slope $\alpha$ indicates that for curved conditioning surfaces the phenomenal flatness is shifted over a distance of $\alpha$ times the conditioning curvature. The fitted relations are (a) $-0.23+0.18 C ; R^{2}=0.99$ (for subject GD), (b) $-0.41+0.18 C ; R^{2}=0.93$ (for subject $\mathrm{MH}$ ), and (c) $-0.15+0.15 C ; R^{2}=0.99$ (for subject $\mathrm{EK}$ ), 


\section{Experiment 2}

It has been shown in experiment 1 that curved surfaces give rise to a haptic aftereffect. It is clear that feeling a surface takes a finite time. We investigated how the aftereffect builds up over time and also investigated whether the aftereffect saturates. We varied the time during which the conditioning surface was touched (conditioning period) and measured the influence of time on the value of the phenomenal flatness and the threshold.

\subsection{Method}

The stimuli and the experimental setup were identical to those in experiment 1 . Three naïve paid subjects participated in this experiment; one was a strongly left-handed male (MZ) and two were strongly right-handed males (TB and $\mathrm{MH}$ ). All subjects were about 20 years old. $\mathrm{MH}$ also participated in the first experiment.

Only three conditioning curvatures were used: $-4,0$, and $4 \mathrm{~m}^{-1}$. The duration of the conditioning period was varied systematically: 2, 5, and $10 \mathrm{~s}$. From pilot experiments this seemed to be the most interesting range, but we were also interested in shorter and longer durations. Because measuring the results for one duration took at least $3 \mathrm{~h}$, we restricted the number of durations. We added a duration of $20 \mathrm{~s}$ for subject TB and durations of $1,20,40$, and $60 \mathrm{~s}$ for subject $\mathrm{MH}$.

A psychometric curve was measured for each combination of conditioning curvature and conditioning period (again by means of nine test surfaces which were presented 15 times).

\subsection{Results}

In figure 5 the influence of the duration of the conditioning period on the magnitude of the aftereffect is shown for subject $\mathrm{MH}$. For the conditioning surfaces of $4 \mathrm{~m}^{-1}$ and $-4 \mathrm{~m}^{-1}$ the phenomenal flatness is plotted against the duration of the conditioning period. We subtracted the shift induced by a flat conditioning surface $\left(\mu_{0}\right)$ from the shift induced by the curved conditioning surfaces because the phenomenal flatness appeared to be liable to small day-to-day variations. The experiment, which took at least $9 \mathrm{~h}$, could not be performed in a single day. So it was split into sessions and for each duration all presentations involving the three conditioning surfaces were measured in the same group of days. Different durations were measured on different days.

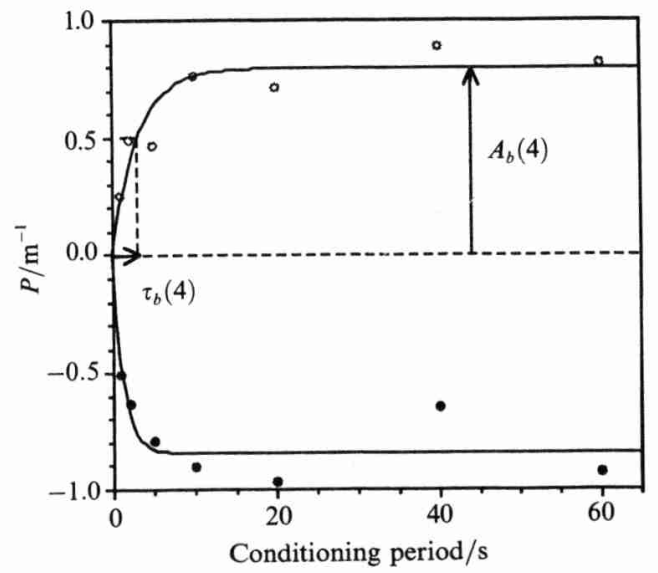

Figure 5. The relation between duration of conditioning period and phenomenal flatness, $P$, in the case of a conditioning curvature of $-4 \mathrm{~m}^{-1}$ (filled circles) and $4 \mathrm{~m}^{-1}$ (open circles), for subject MH. A first-order integrator is fitted: $P(t)=A_{b}\left(1-\mathrm{e}^{-t / \tau_{b}}\right)$. The amplitude $A_{b}$ and the time constant $\tau_{b}$ are indicated only for the conditioning curvature of $4 \mathrm{~m}^{-1}$. The values of the amplitudes $A_{b}(4)$ and $A_{b}(-4)$ and the time constants $\tau_{b}(4)$ and $\tau_{b}(-4)$ are given in table 1 . 
Table 1. Values of $A_{b}$ and $\tau_{b}$ in the case of a conditioning curvature of $-4 \mathrm{~m}^{-1}$ and $4 \mathrm{~m}^{-1}$.

\begin{tabular}{lllll}
\hline Subject & $A_{b}(4) / \mathrm{m}^{-1}$ & $A_{b}(-4) / \mathrm{m}^{-1}$ & $\tau_{b}(4) / \mathrm{s}$ & $\tau_{b}(-4) / \mathrm{s}$ \\
\hline MZ & 0.27 & -0.67 & 1.6 & 1.6 \\
TB & 0.77 & -0.92 & 1.5 & 1.8 \\
MH & 0.79 & -0.85 & 3.3 & 1.3 \\
& & & & \\
\hline
\end{tabular}

Because of the day-to-day variations, the values of the phenomenal flatness could not be compared directly. To correct for this, we subtracted $\mu_{0}$ for each duration. (1)

It can be seen from figure 5 that for both curved conditioning surfaces an increase in the duration results in a larger aftereffect. After a few seconds saturation occurs; the aftereffect does not increase any more. Because of this, we fitted a first-order integrator, $P(t)=A_{b}\left(1-\mathrm{e}^{-t / \tau_{b}}\right)$. The amplitude $A_{b}$ corresponds to the magnitude of the aftereffect at the point of saturation. The time $t$ corresponds to the duration of the conditioning period. The time constant $\tau_{b}$ is a measure of the steepness of the curve; the smaller $\tau_{b}$, the sooner the saturation level is reached. Actually, the time constant is the time at which the magnitude of the aftereffect is $63 \%$ of its maximum $A_{b}$. The time constants and the saturation levels in the case of the convex and concave surfaces are given in table 1. For all subjects the two time constants $\tau_{b}(4)$ and $\tau_{b}(-4)$ are not significantly different. The absolute values of the saturation levels $A_{b}(4)$ and $A_{b}(-4)$ are not significantly different for subjects TB and MH, but are significantly different for MZ.

The thresholds of the psychometric curves did not vary with the duration of the conditioning period or the conditioning surface. They were of about the same magnitude as in experiment 1.

\section{Experiment 3}

When two surfaces are touched sequentially some finite time elapses between the removal of the hand from the first surface and the contact with the second surface. This time interval could influence the magnitude of the aftereffect, although it is also conceivable that the aftereffect is maintained as long as the hand is not stimulated. In experiment 3 we investigated which of these possibilities occurs. We did this by varying the length of the time interval between the conditioning period and the touching of the test surface.

\subsection{Method}

The stimuli and the experimental setup were identical to those in experiments 1 and 2 . Two naïve paid subjects participated in this experiment, MV (24 years) and MB ( 25 years). Both were strongly right-handed females. Three conditioning curvatures were used: $-4,0$, and $4 \mathrm{~m}^{-1}$. The duration of the conditioning period was always $10 \mathrm{~s}$. This time was chosen because we wanted the experiment to take as little time as possible and the magnitude of the aftereffect to be as large as possible. The previous experiment had shown that after a conditioning period of $10 \mathrm{~s}$ the aftereffect did not

(1) A second reason for subtracting this shift was that the magnitude of the aftereffect is not the value of the phenomenal flatness itself, but it is the shift of the phenomenal flatness compared with that in a condition without adaptation. The presentation of a flat conditioning surface is not the same as a condition without adaptation. However, it turned out that $\mu_{0}$ hardly changed with the duration of the conditioning period, whereas the shift induced by the curved conditioning surfaces did change. This indicates that the aftereffect builds up over time and that in the case of a flat conditioning surface the aftereffect is negligible. Apparently, the value of the phenomenal flatness without adaptation is about the same as the constant value of the phenomenal flatness when the conditioning surface is flat. 
increase further. The time interval between the conditioning period and the touching of the test surface was varied: 5,20 , and $40 \mathrm{~s}$. During this interval subjects had to hold their hand in the air in a comfortable manner, but keep their fingers almost straight. They were not allowed to move their fingers during this interval. Because it appeared that the aftereffect had not vanished completely after $40 \mathrm{~s}$, we added a time interval of $80 \mathrm{~s}$ for one subject, namely MV. A psychometric curve was measured for each combination of conditioning curvature and time interval.

\subsection{Results}

In figure 6 the influence of the time interval is shown for subject MV. For the conditioning surfaces of $4 \mathrm{~m}^{-1}$ and $-4 \mathrm{~m}^{-1}$ the phenomenal flatness is plotted against the length of the time interval. The shift induced by a flat conditioning surface is again subtracted. For both conditioning curvatures an increase in the time interval results in a smaller aftereffect. After some period, which is clearly longer than $40 \mathrm{~s}$, the aftereffect will vanish. An exponential decay $P(t)=A_{d} \mathrm{e}^{-t / \tau_{d}}$ is fitted to the data points. The time $t$ corresponds to the length of the time interval. The amplitude $A_{d}$ corresponds to the magnitude of the aftereffect when there is no time interval $(t=0 \mathrm{~s})$. The time constant $\tau_{d}$ is the time at which the magnitude of the aftereffect is $37 \%$ of its starting level $A_{d}$. The time constants and amplitudes in the case of the convex and concave conditioning surfaces are given in table 2 . For both subjects the two time constants $\tau_{d}(4)$ and $\tau_{d}(-4)$ are not significantly different, but the two amplitudes $A_{d}(4)$ and $A_{d}(-4)$ are significantly different.

The thresholds of the psychometric curves did not vary with the length of the time interval and were of about the same magnitude as in experiments 1 and 2 .

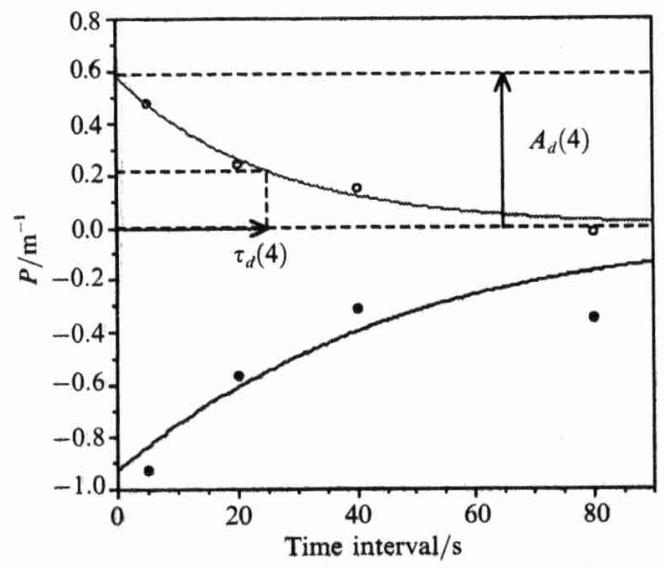

Figure 6. The relation between length of the time interval and phenomenal flatness, $P$, in the case of a conditioning curvature of $-4 \mathrm{~m}^{-1}$ (filled circles) and $4 \mathrm{~m}^{-1}$ (open circles), for subject MV. An exponential decay function is fitted: $P(t)=A_{d} \mathrm{e}^{-t / \tau_{d}}$. The amplitude $A_{d}$ and the time constant $\tau_{d}$ are indicated only for the conditioning curvature of $4 \mathrm{~m}^{-1}$. The values of the amplitudes $A_{d}(4)$ and $A_{d}(-4)$ and the time constants $\tau_{d}(4)$ and $\tau_{d}(-4)$ are given in table 2 .

Table 2. Values of $A_{d}$ and $\tau_{d}$ in the case of a conditioning curvature of $-4 \mathrm{~m}^{-1}$ and $4 \mathrm{~m}^{-1}$.

\begin{tabular}{lllll}
\hline Subject & $A_{d}(4) / \mathrm{m}^{-1}$ & $A_{d}(-4) / \mathrm{m}^{-1}$ & $\tau_{d}(4) / \mathrm{s}$ & $\tau_{d}(-4) / \mathrm{s}$ \\
\hline MZ & 0.58 & -0.96 & 25 & 47 \\
TB & 0.44 & -0.77 & 49 & 36 \\
\hline
\end{tabular}




\section{Discussion}

From experiment 1 we conclude that the phenomenal flatness of a surface is strongly influenced by a previously touched curved surface. The sign of the geometrical curvature (convex/concave) does not necessarily correspond to the convex/concave judgments of a human subject. Thus observers apparently do not have an absolute reference for curvature. We have shown that phenomenal flatness is shifted towards the curvature of the conditioning surface. This aftereffect of curved surfaces is perhaps surprising because most people tend to believe that we need only put our hand on an object to obtain a veridical impression of its shape. On the other hand, many different kinds of aftereffects occur in other modalities. What is unexpected, however, is that the aftereffect appears to be strong, occurs almost immediately, and lasts for a long period. Experiment 1 has shown that after a conditioning period of $5 \mathrm{~s}$ the shift of the phenomenal flatness is about as large as $20 \%$ of the conditioning curvature. A subject may actually judge a sphere with radius $1 \mathrm{~m}$ to be flat! This curvature is greater than the measured threshold of curvature detection by a factor of about 4 . Experiment 2 has shown that after only $2 \mathrm{~s}$ of touching the conditioning surface the aftereffect is already evident. In daily life most objects are touched for at least a second. This means that every time we hold an object the phenomenal flatness is changed. To investigate whether the aftereffect for the condition of a passive hand rapidly decreases or is maintained for a longer period, we roughly estimated the time constant of decay in experiment 3 . The decay of the aftereffect is clearly much slower than the buildup, for both subjects with both conditioning curvatures (by a factor of about 20). So touching a curved object for a short time results in an aftereffect even after a relatively long passive period of $40 \mathrm{~s}$.

As mentioned in section 1, Gibson (1963) measured the magnitude of the aftereffect of vergence for several adaptation periods and recovery periods. He found that for adaptation periods of $12 \mathrm{~s}$ or longer the aftereffect did not increase with time and for recovery periods between $7 \mathrm{~s}$ and $56 \mathrm{~s}$ there was no difference in the magnitude of the aftereffect. These results look quite similar to our findings, though our sensorimotor task was quite different.

Our experiments give rise to many questions. With respect to the time constants for the buildup $\left(\tau_{b}\right)$ and the decay $\left(\tau_{d}\right)$ all we can say is that the values hold for a conditioning surface of $-4 \mathrm{~m}^{-1}$ and $4 \mathrm{~m}^{-1}$. The time constants may be different for other conditioning curvatures. It was shown in experiment 1 , for instance, that the magnitudes of the aftereffect for different conditioning curvatures are different after a conditioning period of $5 \mathrm{~s}$. This could be explained by a fixed time constant $\tau_{b}$ for all conditioning curvatures and an $A_{b}$ that depends on the curvature. It is also conceivable that $A_{b}$ is fixed for all conditioning curvatures whereas $\tau_{b}$ depends on the curvature. Although it would be interesting to investigate the time constants more extensively, this falls outside the scope of this paper.

We measured the time course of the decay of the aftereffect after it had saturated (a conditioning period of $10 \mathrm{~s}$ ). It is also of interest to measure the time course after shorter conditioning periods. If the underlying mechanisms responsible for the aftereffect behave like a simple first-order system we would expect the time constant to be the same for all conditioning periods.

At present the physiological substrate of the aftereffect is unknown. It is known that skin, joint, and muscle receptors adapt, but whether they determine the strength of the aftereffect has yet to be investigated. It is also conceivable that processes in central brain areas play a role, although we do not exactly know how, because these processes are not well documented. More psychophysical and physiological knowledge is needed before we can say which processes are involved in the aftereffect of curved surfaces. 
Because this aftereffect is so strong and fast it is perhaps surprising that we apparently fail to notice it in daily life. However, in all modalities aftereffects typically go unnoticed, though close strutiny may reveal their existence. It is a matter of speculation whether this strong aftereffect has a noticeable influence on normal human actions. Most certainly haptics does not provide us with invariant geometrical information about the curvature of environmental objects.

Acknowledgements. This research was supported by the Netherlands Organization of Scientific Research (NWO).

\section{References}

Abbott E, 1914 "The effect of adaptation on the temperature limen" Psychological Monographs $16(68) 1-36$

Colheart M, 1971 "Visual feature-analyzers and after-effects of tilt and curvature" Psychological Review 78 114-121

Coren S, 1993 The Left-hander Syndrome (New York: Vintage Books)

Davidson P W, 1972 "Haptic judgements of curvature by blind and sighted humans" Journal of Experimental Psychology 93 43-55

Gibson J J, 1933 "Adaptation, after-effect and contrast in the perception of curved lines" Journal of Experimental Psychology 16 1-31

Gibson J J, 1937 "Adaptation with negative after-effect" Psychological Review $44222-244$

Gibson J J, 1963 "An after-effect in haptic space perception" Quarterly Journal of Experimental Psychology $15145-154$

Hahn J F, 1966 "Vibrotactile adaptation and recovery measured by two methods" Journal of Experimental Psychology 71 655-658

Hazlewood V, 1971 "A note on failure to find a tactile motion aftereffect" Australian Journal of Psychology 23 59-62

Hollins M, Favorov O, 1994 "The tactile movement aftereffect" Somatosensory and Motor Research $11153-162$

Hunter I M L, 1954 "Tactile-kinaesthetic perception of straightness in blind and sighted humans" Quarterly Journal of Experimental Psychology $6149-154$

Koehler W, Dinnerstein D, 1947 "Figural after-effects in kinesthesis", in Miscellanea Psychologica Albert Michotte Ed. Intitut Supérieur de Philosophie (Louvain: Imprimerie St-Alphonse) pp $196-220$

Nafe J P, Wagoner K S, 1941 "The nature of pressure adaptation" Journal of General Psychology $25323-351$

Press W H, Flannery B P, Teukolsky S A, Vetterling W T, 1988 Numerical Recipes in C (Cambridge: Cambridge University Press)

Thalman W A, 1922 "The after-effect of movement in the sense of touch" American Journal of Psychology $33268-276$

Walker J T, 1977 "Orientation-contingent tactual size aftereffects" Perception \& Psychophysics 22 $563-570$

Walker J T, Shea K S A, 1974 "A tactual size aftereffect contingent on hand position" Journal of Experimental Psychology $103668-674$

Wohlgemuth A, 1911 "On the after-effect of seen movement" British Journal of Psychology, Monograph Supplement 1 